REVUE DE L'INSTITUT

FRANÇAIS D'HISTOIRE

EN ALLEMAGNE

\section{Revue de I'IFHA}

Revue de l'Institut français d'histoire en Allemagne

$1 \mid 2009$

IFHA 1

\title{
Des chemins de la connaissance historique. À propos de l'ouvrage collectif Wege der Gesellschaftsgeschichte
}

Jochen Hoock

\section{(2) OpenEdition \\ Journals}

Édition électronique

URL : http://journals.openedition.org/ifha/345

DOI : 10.4000/ifha.345

ISSN : 2198-8943

Éditeur

IFRA - Institut franco-allemand (sciences historiques et sociales)

Édition imprimée

Date de publication : 30 septembre 2009

Pagination : 126-132

ISSN : 2190-0078

\section{Référence électronique}

Jochen Hoock, « Des chemins de la connaissance historique. À propos de l'ouvrage collectif Wege der Gesellschaftsgeschichte», Revue de l'IFHA [En ligne], 1 | 2009, mis en ligne le 07 février 2013, consulté le 03 mai 2019. URL : http://journals.openedition.org/ifha/345 ; DOI : 10.4000/ifha.345

Ce document a été généré automatiquement le 3 mai 2019.

(CIFHA 


\title{
Des chemins de la connaissance historique. À propos de l'ouvrage collectif Wege der Gesellschaftsgeschichte
}

\author{
Jochen Hoock
}

1 Il y a trente ans, le terme de Gesellschaftsgeschichte désignait autant un choix stratégique qu'une approche méthodologique face au passé récent des sociétés européennes et de la société allemande en particulier ${ }^{1}$. Dans un essai repris par Hans-Ulrich Wehler dans un choix de textes sur les rapports entre histoire et sociologie ${ }^{2}$, Eric Hobsbawm soulignait à la fois l'autonomie de la démarche historienne et son ouverture aux approches multiples des sciences sociales systématiques tout en affirmant sa fonction essentiellement critique. L'« ouverture critique » fut ainsi, à défaut d'une programmatique étroite, le maître-mot lors de la création en 1975 de la revue Geschichte und Gesellschaft, dont le succès fut dans les trois décennies suivantes largement assimilé à ce qu'il est convenu d'appeler l'« École de Bielefeld ».

Le présent volume, en dressant le tableau des chemins ouverts et empruntés par ce que les familiers commencerent très tôt à désigner sous le sigle " GG », se veut, conformément à la démarche initiale, à la fois un bilan et un projet d'avenir pour lequel le changement de paradigme ou, plus simplement, d'orientation devient objet d'un dialogue suivi, conscient de ce qu'il faut bien appeler l'épreuve du temps. Une référence aussi implicite que constante est celle à l'œuvre de Hans-Ulrich Wehler, dont les cinq volumes de la Deutsche Gesellschaftsgeschichte se confondent, ne seraient-ce que par leurs dates de parution, avec la période prise en considération. L'interrogation porte sur la constance et l'évolution d'un questionnaire dont la pertinence est confrontée à l'évolution de son objet et des prises de position que celle-ci a engendrées.

Rétrospectivement, le tournant que représentait Geschichte und Gesellschaft peut être assimilé à un tournant analytique qui, par ses choix méthodologiques, dépasse l'histoire sociale en anticipant sur d'autres changements - la référence bielefeldoise cachant, 
comme le souligne Jürgen Kocka dans sa contribution qui ouvre le volume, la diversité des courants critiques de la fin des années 1960, qui allaient de l'histoire du temps présent, de la linguistique structurale et de l'histoire du droit à celle de l'art et des représentations collectives - tous motivés, jusque dans les querelles méthodologiques, par « un passé qui ne passait pas »... Plus que ne le laisse entrevoir les filiations justement relevées par Jürgen Kocka, citant parmi tant d'autres les noms de Hans Herzfeld, Gerhard A. Ritter ou Hans Rosenberg, ce qui compte, ce sont les doutes et les ambivalences qui par moment s'apparentent à une véritable quête identitaire, à laquelle n'échappèrent pas les débats les plus abstraits comme le montre, pour ne citer qu'un exemple, la réception de l'œuvre de Max Weber qu'accompagnait la réévaluation de l'homme politique par W. J. Mommsen ${ }^{3}$ . La rigueur de la problématique de la Gesellschaftsgeschichte, surtout dans la version qu'en a donnée Hans-Ulrich Wehler, n'a d'équivalent que la tourmente dont elle était issue. L'élargissement et les développements successifs qu'esquisse Jürgen Kocka apparaissent, dans cette perspective, surtout comme des réponses reflétant la vitalité d'un projet qui a sollicité dès le départ les contributions les plus diverses quitte à les assimiler et à les transformer.

4 Parmi ces défis, celui de la sociologie historique est sans doute le plus grand parce que le plus proche de la dimension réflexive de la problématique initiale, dont Jürgen Osterhammel relève, non sans un certain regret, l'« acuité provocante » (p. 101). D'autres disciplines, comme la science politique, ont occupé par moment la place d'une concurrente dont les traits normatifs ne heurtaient pas seulement les intérêts disciplinaires des historiens. La différenciation des domaines de recherches, dont les Bindestrichsoziologien donnaient l'exemple, devenaient vers la fin des années 1970 le moteur d'un "partenariat antagoniste » (Klaus von Beyme), pour lequel les divergences théoriques se situaient au-delà des frontières disciplinaires (p. 43). La coexistence entre théorie critique, théorie des systèmes et des approches plus traditionnellement herméneutiques caractérisait alors la situation dans maintes facultés de philosophie, comme par exemple celle de Heidelberg, et n'épargnait guère l'évolution que connaissaient des créations comme Bielefeld, où la «science historique » se voulait, à travers la nouvelle Fakultät für Geschichtswissenschaft (faculté d'histoire), d'entrée de jeu pluridisciplinaire. L'histoire s'affirmait en cessant d'être un champ clos et en rompant avec une périodisation traditionnelle, ignorant les temporalités et les régimes d'historicité. Geschichte und Gesellschaft était de ce fait redevable autant aux constellations de la « vieille faculté de philosophie » qu'au rôle, marginal seulement en apparence, des Arbeitskreise (cercles de travail) et Institute qui élargissaient le champ universitaire ${ }^{4}$. L'intensité de la querelle du positivisme (Positivismusstreit) ne devait pas nuire à la naissance d'un pluralisme méthodologique qui n'ignorait pas les subtilités d'un marxisme dissident transformé par la théorie de croissance et d'un "formalisme " sensible à la théorie des jeux. Un exemple frappant de la capacité d'intégration de cette conscience clivée de l'historiographie de la fin des années 1970 est la réception de la gender history sous la forme de Frauen- und Geschlechtergeschichte (" histoire des femmes et du genre »), dont Gisela Bock donne ici une analyse particulièrement pertinente (p. 45 suiv.).

Malgré les controverses théoriques, le changement d'expérience intergénérationnel restait un facteur important dans la façon dont on abordait le passé. L'incommunicabilité de l'expérience, longtemps l'un des non-dits du débat entre historiens, formait à certains égards la véritable trame d'un travail qui dépassait la "maîtrise du passé». La contribution de Dieter Langewiesche, consacrée à la « réécriture de l'histoire », relève ici 
la contribution fondamentale de Reinhart Koselleck tout en analysant dans une perspective plus large le rôle de l'histoire sociale dans l'auto-affirmation des sociétés européennes, la « réalité historique » d'un moment ne se révélant qu'à travers l'épaisseur de la distance temporelle ouvrant des perspectives inaccessibles aux contemporains (p. 67 suiv.). Là où $\mathrm{D}$. Langewiesche souligne la dimension anthropologique du débat des années 1970, Jürgen Osterhammel revient dans une contribution également dense sur l'usage spécifique de l'offre théorique des sciences sociales par le groupe de Bielefeld (p. 81 suiv.). En distinguant trois aspects dans la réception des débats théoriques (rigueur conceptuelle, usage restrictif et partiel, champ d'application limité), J. Osterhammel dégage les éléments efficaces d'une approche que la critique a souvent assimilée à une version germanique du "plan-type » attribué à l'école labroussienne. Revenant sur les implications de la théorie des temps historiques pour l'histoire sociale et la contribution de R. Koselleck, il reprend, à partir des contributions à la Gesellschaftsgeschichte, les avances de l'analyse des processus historiques et sociaux dans leurs dimensions spatiotemporelles. Dans quelle mesure servent-ils le désir d'explication causale qui a motivé la résistance des tenants de la Gesellschaftsgeschichte à l'approche descriptive de l'histoire culturelle ? Les réponses ramènent, par-delà l'opposition traditionnelle entre explication et compréhension, à la théorie de l'action et au problème d'incertitude. Le débat est ainsi ouvert, laissant derrière lui les certitudes d'un moment en reprenant le débat engagé il y a trente ans.

Or, parmi les idées forces du début des années 1970, la théorie de la modernisation occupait une place de choix. Ses multiples variantes se distinguaient non seulement par leurs connotations idéologiques, mais aussi par leur capacité à offrir des modèles d'interprétation répondant aux critères d'une démarche historienne ${ }^{5}$. C'est à ces tentatives de transposition de tels concepts que s'intéresse Paul Nolte en dressant le bilan d'une historiographie centrée autour de la période de transition du XIXe siècle lue comme le prélude du siècle suivant. Faut-il maintenir l'idée d'un siècle des révolutions ou céder à une vision plus ambivalente de la modernité ? Plaidant pour l'abandon d'une conception par trop statique de la modernité en intégrant les coûts et les pertes d'un processus plus que complexe, Nolte fait surtout ressortir les déficits du master narrative axé sur une seule période de transformation et longtemps enfermé dans des catégories post-hégéliennes (p. 110 suiv.). Au lieu de s'attacher exclusivement au syndrome structurel de la modernité, il serait utile de porter le regard sur le processus de la modernisation et ses multiples temporalités.

7 Parmi les choix méthodologiques que P. Nolte évoque, les différents tournants qu'ont connus les années 1990 occupent une place de choix. Leur dynamique propre est l'objet d'une analyse de Christoph Conrad qui focalise pour l'essentiel sur le passage de l'histoire sociale à l'histoire culturelle telle que l'a définie le cultural turn (p. 133 suiv.). L'analyse séduit par sa dimension lexicographique et son caractère international. L'évaluation du «tournant critique» des Annales, dont la référence aux sciences sociales est curieusement passée sous silence, permet au lecteur français de se faire une idée de la difficulté de prendre toute la mesure du phénomène. L'explication convainc en revanche par la distinction très claire entre une approche externaliste et internaliste qui relève à juste titre la rupture générationnelle qui, plus que toute autre, a surtout frappé la génération socialisée aux cours des années 1960 (p. 154). Faudrait-il alors interpréter les chemins de la connaissance historique outre-Rhin tels que les a dessinés la Gesellschaftsgeschichte comme l'expression d'un traumatisme tel que le supposait le 
concept d'une "maitrise du passé »? Quelle importance accorder à la lecture de ces changements à travers l'interprétation qu'en donne l'un de ses principaux protagonistes, à savoir Hans-Ulrich Wehler? Une fois intégrées les multiples différenciations aux frontières de l'histoire sociale comme l'influence croissante de l'anthropologie, du gender et de la micro-storia, il reste les grands bouleversements dans la perception et l'expérience du monde, la présence de l'altérité au quotidien et sa gestion au jour le jour, en somme : une globalisation assumée au quotidien et génératrice d'une réévaluation de la place centrale que l'historiographie, y compris la Gesellschaftsgeschichte, avait jusqu'ici accordée aux mondes européens.

8 Cet élargissement de l'horizon d'expérience face à un monde fini est sous-jacent à l'ensemble des contributions. Ulrike Freitag reprend cette problématique pour le monde arabe sans prétendre développer des "paramètres de la modernité » des civilisations extra-européennes comme J. Osterhammel les a proposés pour la Chine (p. 161 suiv.). Le résultat est une sorte d'heuristique pour une histoire des sociétés orientales plaidant pour une immanence de la chronologie des adaptations au contact culturel. Plus proche d'une logique de paths of development au sens traditionnel du terme est par contre le récit que donne Hans-Jürgen Puhle du «syndrome atlantique » et des différentes formes de globalisation qui s'y rattachent (p. 179 suiv.). Son intérêt réside dans la typologie de constellations qui caractérisent et "forment» les interactions transatlantiques. Analysant les cas des deux Amériques dans leurs rapports avec le continent européen, H.J. Puhle fait surtout ressortir l'emboîtement des espaces de développement et leur articulation régionale et ethnique extrêmement différenciée s'opposant à une quelconque path dependency qui imposerait un modèle ou une réponse uniques.

9 Les deux contributions qui s'attachent plus spécifiquement à l'histoire économique et sociale expriment une même réserve à l'égard d'un modèle de développement linéaire. Pour Werner Abelshauser, nous sommes actuellement témoins et protagonistes d'un double changement de paradigme (p. 203 suiv.). Le premier remplace la notion de « révolution industrielle » par un long cheminement de la modernité, le deuxième passe de la prédominance de la production matérielle à celle de l'immatériel ou, plus exactement, à celle de l'organisation d'une production de savoirs créant les conditions d'une offre élastique d'innovations scientifiques et technologiques. Dans le premier cas, tout porte à reconsidérer le poids de changements technologiques face à la lente mise en place des dispositions institutionnelles et intellectuelles préparant et accompagnant la première phase de l'industrialisation. La principale caractéristique de la période préindustrielle serait au contraire la capacité de garantir la « continuité » d'institutions modernes face au danger que constitueraient des ruptures dans le développement. La "révolution industrielle» doit donc être vue comme le point culminant d'un long développement plutôt que comme un point de départ radicalement neuf ${ }^{6}$. Dans le deuxième cas, il s'agit de comprendre comment les sociétés de la fin du XIXe siècle se sont montrées capables de créer les cadres institutionnels nécessaires pour rapprocher la production des savoirs et l'évolution des forces productives, afin de mobiliser un potentiel de productivité jusque-là inconnu. Illustrée par le cas allemand, l'argumentation conforte le modèle d'un corporate investment que $\mathrm{W}$. Abelshauser a développé pour la phase de "reconstruction » du capitalisme rhénan dans la période de l'après-guerre.

10 Cette hypothèse est prise à rebours par la contribution de Charles Tilly s'interrogeant sur la validité d'un « modèle allemand » du développement économique. Le débat qui tourne 
autour des fondamentaux de l'histoire économique depuis l'intervention de Douglass C. North reflète de manière la plus pertinente l'esprit qui a animé le groupe autour de Geschichte und Gesellschaft et il n'est donc pas étonnant que le volume reprenne in fine des thèmes d'actualité comme celui d'une politique du « juste milieu » de Manfred G. Schmidt ou de la démesure financière et budgétaire analysée par Hans-Peter Ullmann pour revenir finalement au problème de l'inégalité sociale discuté par Klaus Tenfelde.

11 Avec l'analyse de K. Tenfelde, qui comme Charles Tilly met l'accent sur les développements régionaux dans une économie globale, resurgit la dimension programmatique de l'entreprise de la Gesellschaftsgeschichte telle que J. Kocka l'avait soulignée fortement dans son chapitre introductif. Elle implique, comme le relève Christoph Conrad, la critique explicite des paradigmes dominants. Mais la meilleure définition d'une telle démarche est sans doute celle de Hans-Ulrich Wehler affirmant que la bonne méthode est celle qui travaille à son dépassement.

\section{NOTES}

1. Compte rendu de: Jürgen Osterhammel, Dieter Langewiesche, Paul Nolte (dir.), Wege der Gesellschaftsgeschichte, Göttingen: Vandenhoeck \& Ruprecht (Geschichte und Gesellschaft, Sonderheft, 22), 2006, 294 p., 39,90 Ä.

2. Eric Hobsbawm, « From social history to the history of society » in : Hans-Ulrich Wehler (dir.), Geschichte und Soziologie, Köln : Kiepenheuer \& Witsch, 1972, p. 331-353.

3. Wolfgang J. Mommsen, Max Weber und die deutsche Politik, 1890-1920, Tübingen: Mohr, 1959; Mommsen était un des co-fondateurs de Geschichte und Gesellschaft.

4. Citons ici seulement le Arbeitskreis für Sozialgeschichte de Heidelberg (Brunner, Conze), la Stelle für Sozialforschung à Dortmund (Schelsky), le groupe autour de René König à Cologne, le Otto-SuhrInstitut à Berlin ...

5. Hans-Ulrich Wehler, Modernisierungstheorie und Geschichte, Göttingen: Vandenhoeck \& Ruprecht, 1975.

6. Voir aussi Jochen Hoock, «Professional ethics and commercial rationality at the Beginning of the Modern Era " in: Margaret Jacob, Catherine Secretan (dir.), The Self-representation of Early Modern Capitalists, New York : Palgrave Macmillan, 2008, p. 147-160.

\section{AUTEUR}

\section{JOCHEN HOOCK}

Jochen Hoock est professeur émérite d'histoire moderne à l'Université Denis-Diderot Paris VII et président de la Fondation pour la Science. 